\section{Predicting the Impact of Contact Precautions on Methicillin-Resistant Staphylococcus aureus Outcomes: Caution Up Ahead}

To the Editor-We read with interest the article by Lee et al $^{1}$ that was recently published in the journal. The authors utilized a novel agent-based mathematical model to explore how variable application of contact precautions in nursing homes (NHs) impacts the prevalence and acquisition of methicillinresistant Staphylococcus aureus (MRSA) in regional healthcare facilities. In the authors' model, targeted application of contact precautions to residents with clinically apparent infection prevented approximately 100 MRSA acquisition events in study NHs over a 5-year period and had a negligible impact on MRSA prevalence in regional hospitals. Wider application to all residents known to be colonized with MRSA (approximately $25 \%$ of the $\mathrm{NH}$ population in the study) avoided approximately 5,000 MRSA acquisition events in study $\mathrm{NHs}$ and led to a nearly $6 \%$ relative reduction in the prevalence of MRSA in regional hospitals over the same time period.

The authors are careful to point out that mathematical models necessarily simplify real-world phenomena and that the utility of using contact precautions to prevent MRSA acquisition in NHs needs to be validated by actual trials. However, we believe there are several aspects of this infection control strategy that deserve careful consideration before embarking on studies in NHs. First, while there is reasonably good evidence that MRSA colonization has a significant impact on health outcomes in hospitalized patients, there is less evidence of a similar effect on the health outcomes of $\mathrm{NH}$ residents who are not hospitalized. Additional studies examining the impact of MRSA colonization on NH resident outcomes are needed to quantify the clinical benefit NH residents would accrue through avoidance of MRSA colonization. While the primary benefit of expanded use of contact precautions in NHs would be through regional reductions in the prevalence of MRSA, the large uncertainty surrounding estimates of this benefit (in the study of Lee and colleagues, relative reductions in the prevalence of MRSA in hospitals ranged from 0.3 to $17.0 \% ;^{1}$ see their Table 2) raises concern about such studies being adequately powered to detect meaningful reductions in this marker.

A second concern of ours centers on the efficacy and consequences of contact precautions in NHs. Many NHs employ modified contact precautions, in which staff utilize gloves and gowns during direct care activities and liberalize resident opportunities for social interaction. Lee and colleagues recommend a more aggressive version of contact precautions that requires affected residents to wear gowns and gloves outside their room. Residents and visitors could interpret the need for gloves and gowns as a sign of "contamination," and identification of MRSA colonization in a long-term-stay resident essentially would represent a "life sentence." Accordingly, we believe that application of this new stigmatizing form of contact precautions should require especially solid data to indicate efficacy. However, we are unaware of any studies examining the impact of any form of contact precautions in NHs. Moreover, the resources required to maintain this more aggressive form of contact precautions for a quarter of the $\mathrm{NH}$ population are not currently available in most facilities. Finally, while the authors appropriately cite studies that have raised concerns about the adverse health and psychological consequences of contact isolation in hospital settings, there is reason to believe that the form of contact precautions advocated by Lee and colleagues would amplify these adverse consequences. We believe that more research on the benefits of, consequences of, and alternatives to contact precautions are needed before pursuit of largescale studies of its impact on MRSA acquisition in NHs.

A final concern about the authors' approach centers on the assumption that MRSA acquisition and, by extension, transmission rates in NHs are homogeneous. A previous publication by the same authors found that residents healthy enough to participate in group activities incur less risk of MRSA. ${ }^{2}$ Moreover, Furuno et $\mathrm{al}^{3}$ found marked differences in MRSA transmission rates in rehabilitation versus residential stay wards, and Bradley et $\mathrm{al}^{4}$ found little evidence of ongoing MRSA transmission between $\mathrm{NH}$ residents who shared rooms. These data highlight how little we know about the routes and rates of MRSA transmission in NHs, and we suggest that considerably more effort be placed on understanding these mechanisms before embarking on a study of contact precautions in NHs.

In summary, Lee and colleagues are to be congratulated for highlighting the important influence NH infection control practices can have on regional rates of MRSA. However, we believe that studies of the wider application of contact precautions in NHs should be avoided until we have a better understanding of the effectiveness and consequences of these practices and have more knowledge of the mechanisms by which residents acquire MRSA in NHs and the individual consequences of this condition.

\section{ACKNOWLEDGMENTS}

Potential conflicts of interest. All authors report no conflicts of interest relevant to this article. All authors submitted the ICMJE Form for Disclosure of Potential Conflicts of Interest, and the conflicts that the editors consider relevant to this article are disclosed here.

Christopher J. Crnich, MD, MS, FACP; Paul Drinka, $\mathrm{MD}^{2}$ 
Affiliations: 1. Division of Infectious Diseases, University of Wisconsin School of Medicine and Public Health, Madison, Wisconsin; 2. Internal Medicine/Geriatrics, University of Wisconsin, Madison, and Medical College of Wisconsin, Milwaukee, Wisconsin.

Address correspondence to Christopher J. Crnich, MD, MS, FACP, Division of Infectious Diseases, University of Wisconsin School of Medicine and Public Health, 1685 Highland Avenue, MFCB 5217, Madison, WI 53705 (cjc@medicine.wisc.edu, pauldrinka@sbcglobal.net). Infect Control Hosp Epidemiol 2013;34(6):646-647

(C) 2013 by The Society for Healthcare Epidemiology of America. All rights reserved. 0899-823X/2013/3406-0019\$15.00. DOI: 10.1086/670644

\section{REFERENCES}

1. Lee BY, Singh A, Bartsch SM, et al. The potential regional impact of contact precaution use in nursing homes to control methicillinresistant Staphylococcus aureus. Infect Control Hosp Epidemiol 2013;34(2):151-160.

2. Murphy CR, Quan V, Kim D. Nursing home characteristics associated with methicillin-resistant Staphylococcus aureus (MRSA) burden and transmission. BMC Infect Dis 2012;12:269.

3. Furuno JJ, Shurland SM, Zhan M, et al. Comparison of methicillin-resistant Staphylococcus aureus acquisition among rehabilitation and nursing home residents. Infect Control Hosp Epidemiol 2011;32(3):244-249.

4. Bradley SF, Terpenning MS, Ramsey MA, et al. Methicillin-resistant Staphylococcus aureus: colonization and infection in a longterm care facility. Ann Intern Med 1991;115(6):417-422.

\section{Reply to Crnich and Drinka}

To the Editor-We appreciate the insightful comments of Crnich and Drinka ${ }^{1}$ about our recently published study. ${ }^{2}$ They raise a number a number of excellent points about employing contact precautions in nursing homes (NHs), emphasizing that the efficacy and consequences of such a practice are not yet fully understood.

Our simulation modeling study, using RHEA (Regional Healthcare Ecosystem Analyst), ${ }^{3-6}$ aimed to galvanize and further the discussion on using contact precautions in NHs, not to establish a final recommendation. So Crnich and Drinka's letter is one of the desired results of our study. As stated in the discussion, we fully agree that caution about use of contact precautions in NHs is important given the risk for isolation or stigma when a home environment is essential. However, we also note that some NHs currently employ contact precautions, especially those that care for higher-risk postacute patients with relatively short (approximately 2 weeks) lengths of stay. ${ }^{7,8}$ In addition, contact precautions may be a viable and necessary solution to curb outbreaks.

The purpose of our article was to quantify the potential effects of contact precautions in NHs. This estimate may be helpful in weighing the advantages and disadvantages of their use in certain $\mathrm{NH}$ populations or under certain conditions, such as outbreaks. Perhaps most importantly, it provides an estimate against which the effectiveness of alternative inter- ventions can be compared. Such estimates are hard to obtain through epidemiologic studies, and this model may provide early insight into the magnitude of benefit while we await confirmatory studies.

Simulation models like RHEA offer a much less expensive and much safer arena to evaluate different large-scale possibilities before unveiling them in the real world. Another benefit of modeling is to identify current gaps in data and understanding so as to guide future studies and data collection. For example, varying parameters such as contact precaution efficacy and compliance can delineate their potential impact and thus help prioritize data collection for researchers, policy makers, administrators, and funders. Of note, in response to Crnich and Drinka's comments, our RHEA model does not assume that methicillin-resistant Staphylococcus aureus (MRSA) acquisition and transmission rates are homogeneous across NHs. Only the mixing patterns of patients within each $\mathrm{NH}$ were homogeneous.

In addition, modeling is an iterative progressive task. Initial models contain assumptions and simplifications. Experts such as Crnich and Drinka offer comments and suggestions that can help guide the next round of refinements to the model. With each progressive modeling round along with concomitant data collection and clinical studies, the field moves closer and closer to a specific set of recommendations. The final preferred $\mathrm{NH}$ intervention may be adaptation of some current acute care contact precaution procedures that accounts for the challenges elucidated by Crnich and Drinka, such as the behavioral and psychological issues associated with glove and gown use. A combination of active dialogue, planning, and innovative approaches will be the way to tackle the important problem of MRSA and other infectious diseases in NHs.

\section{ACKNOWLEDGMENTS}

Financial support. This study was supported by the National Institute of General Medical Sciences Models of Infectious Disease Agent Study (MIDAS) grants 5U54GM088491-02 and 1U01GM076672 and the Pennsylvania Department of Health. This project was also funded under contract HHSA29020050033I from the Agency for Healthcare Research and Quality, US Department of Health and Human Services, as part of the Developing Evidence to Inform Decisions about Effectiveness (DEcIDE) program.

Potential conflicts of interest. All authors report no conflicts of interest relevant to this article. All authors submitted the ICMJE Form for Disclosure of Potential Conflicts of Interest, and the conflicts that the editors consider relevant to this article are disclosed here.

\section{Bruce Y. Lee, MD, MBA; ${ }^{1}$ Sarah M. Bartsch, MPH; ${ }^{1}$} Susan S. Huang, MD, $\mathrm{MPH}^{2}$

Affiliations: 1. Public Health Computational and Operations Research (PHICOR), University of Pittsburgh, Pittsburgh, Pennsylvania; 2. University of California, Irvine, California.

Address correspondence to Bruce Y. Lee, MD, MBA, PHICOR, University of Pittsburgh, 200 Meyran Avenue, Suite 200, Pittsburgh, PA 15213 (byl1@pitt.edu).

The authors of this report are responsible for its content. Statements in 